\title{
CHANDRA-LETGS X-ray observations of Capella
}

\section{Temperature, density and abundance diagnostics}

\author{
R. Mewe ${ }^{1}$, A. J. J. Raassen ${ }^{1,2}$, J. J. Drake ${ }^{3}$, J. S. Kaastra ${ }^{1}$, R. L. J. van der Meer ${ }^{1}$, and D. Porquet ${ }^{4}$ \\ 1 Space Research Organization Netherlands (SRON), Sorbonnelaan 2, 3584 CA Utrecht, The Netherlands \\ 2 Astronomical Institute "Anton Pannekoek", Kruislaan 403, 1098 SJ Amsterdam, The Netherlands \\ 3 Smithsonian Astrophysical Observatory, 60 Garden Street, Cambridge, MA 02138, USA \\ 4 CEA/DSM/DAPNIA, Service d'Astrophysique, CEA Saclay, 91191 Gif-sur-Yvette Cedex, France
}

Received 12 October 2000 / Accepted 15 December 2000

\begin{abstract}
We report an analysis of the X-ray spectrum of Capella from 6 to $175 \AA$ obtained with the Low Energy Transmission Grating Spectrometer (LETGS) on board of the X-ray space observatory CHANDRA. Many emission line features appear that can be resolved much better as compared to former instruments (EUVE and ASCA). Coronal electron densities $\left(n_{\mathrm{e}}\right)$ and temperatures $(T)$ of brightly emitting regions are constrained by an analysis of ratios of density- and temperature-sensitive lines of helium-like ions and highly ionized iron atoms. Lines emitted by e.g., O VII \& VIII, Mg X-XII, Si XII-XIV, Fe IX, X \& XV-XXIII are used to derive T. Line ratios in the helium-like triplets of $\mathrm{C} \mathrm{V}, \mathrm{N}$ VI, O VII, Mg XI, and Si XIII yield $T$ in the range 0.5-10 MK, and $n_{\mathrm{e}}$ in the range $10^{9}-10^{13} \mathrm{~cm}^{-3}$. The Fe IX/X ratio yields $T \simeq 0.9 \mathrm{MK}$, while lines from Fe XVIII to XXII give $T \sim 6-10$ MK. Flux ratios of Fe XX-XXII lines indicate for the electron densities an upper limit in the range $n_{\mathrm{e}} \lesssim(2-5) 10^{12} \mathrm{~cm}^{-3}$. From line ratios of Fe XVII and Fe XVIII we derive constraints on the optical depth $\tau$ of $\sim 1-1.5$. An emission measure distribution is derived from Fe line intensities. Results for element abundances (relative to Fe) from a 4-T model are: $\mathrm{O}$ and $\mathrm{Ne} / \mathrm{Fe}$ about solar, $\mathrm{N}, \mathrm{Mg}$ and $\mathrm{Si} / \mathrm{Fe} \sim 2 \times$ solar. The results for $T$ and $n_{\mathrm{e}}$ are described in terms of quasi-static coronal loop models and it is shown that the X-ray emission originates from compact structures much smaller than the stellar radii.
\end{abstract}

Key words. stars: individual: Capella - stars: coronae - stars: late-type - stars: activity - X-rays: stars atomic processes vspace-1mm

\section{Introduction}

The G1 III + G8/K0 III binary Capella with a 104.0233day orbit at a distance of $13 \mathrm{pc}$ (Strassmeier et al. 1993) is one of the brightest non-degenerate stellar Xray sources. Observations by Linsky et al. (1998) with the GHRS spectrometer on the Hubble Space Telescope (HST) on Sept. 1995 and on April 1996 indicate that the optically brightest (by 0.15 ) and more fastly rotating $\left(v \sin i=36 \mathrm{~km} \mathrm{~s}^{-1}\right.$ ) G1 component (mass $2.6 M_{\odot}$, radius $9.2 R_{\odot}$ ) is about $3-10$ times brighter in the chromospheric and transition-region UV lines than the slowly rotating $\left(v \sin i=3 \mathrm{~km} \mathrm{~s}^{-1}\right) \mathrm{G} 8 / \mathrm{K} 0 \operatorname{star}\left(2.7 M_{\odot}, 12.2 R_{\odot}\right)$. The Sept. 1995 observations showed that the coronae around the G1 and G8 stars were equally bright in the hot $\left(10^{7} \mathrm{~K}\right)$ coronal Fe XXI $1354 \AA$ line, but recent observations with the HST STIS by Johnson et al. (2001) on October 1999 indicate that the G8 corona was at least 5 times fainter than the G1 corona in the Fe XXI line, the latter having

Send offprint requests to: R. Mewe, e-mail: R.Mewe@sron.nl changed relative to the Sept. 1995 observations by only $50 \%$ or less. These results suggest that the hot corona of the G8 star is quite strongly variable whereas that of the G1 is not.

Due to the coronal structure of Capella its spectrum is rich in emission lines in the soft X-ray region and has been well studied by many earlier instruments with lower spectral resolution (for references see, e.g., Brinkman et al. 2000). The spectra of Capella obtained with the LETGS are of high quality and agree with the pre-flight calculated instrumental spectral resolution (cf. Brinkman et al. 2000). The wavelength region of the LETGS between 6 $42 \AA$ contains a number of helium-like line "triplets" from Si XIII to $\mathrm{C} \mathrm{V}$ which provide density as well as temperature diagnostics for plasmas with temperatures in the range $1-10 \mathrm{MK}$ and densities in the range $10^{8}-10^{13} \mathrm{~cm}^{-3}$. The long-wavelength region between $90-140 \AA$ contains a series of lines from $2 \ell-2 \ell^{\prime}$ transitions in Fe XVIII-Fe XXIII which provide density and temperature diagnostics for relatively hot $(\gtrsim 5 \mathrm{MK})$ and dense $\left(\gtrsim 10^{12} \mathrm{~cm}^{-3}\right)$ plasmas (e.g., Mewe et al. 1985, 1991; Brickhouse et al. 1995). From 

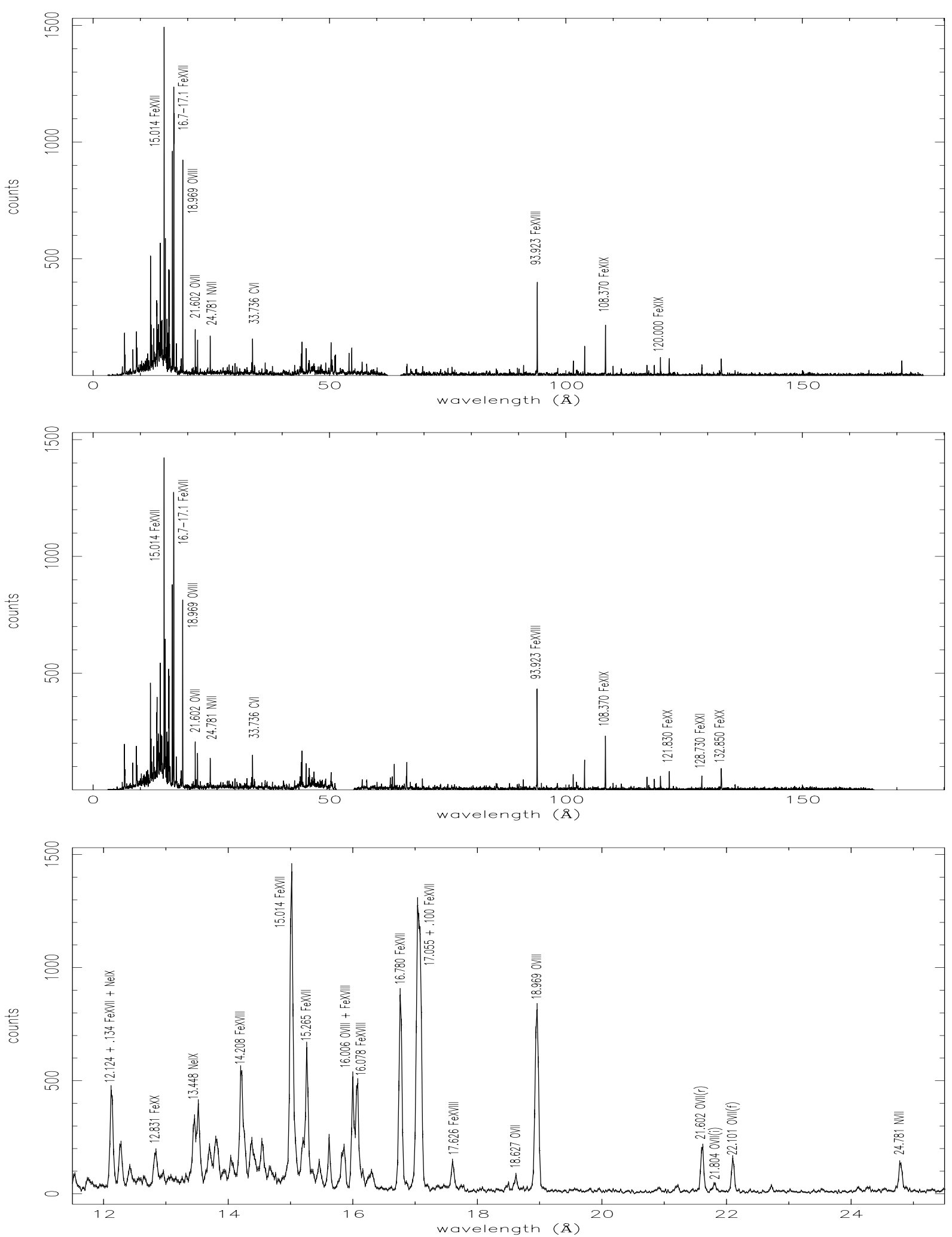

Fig. 1. CHANDRA-LETGS spectrum of Capella observed on 9 Nov. 1999 in +1 order (top), in -1 order (middle), and detail of -1 order spectrum between $12-25 \AA$ (bottom). Spectra are corrected for background. Several prominent lines are labelled with the emitting ions

EUVE spectra of Capella, Dupree et al. (1993), Schrijver et al. (1995), and Brickhouse (1996) have derived electron densities $n_{\mathrm{e}}$ in the range $10^{12}-10^{13} \mathrm{~cm}^{-3}$ at a tempera- ture $T \sim 10$ MK. More recently, Brickhouse et al. (2000) have analyzed the coronal temperature and abundance structure of the coronae of Capella based on EUVE and 
Table 1. Strongest non-iron lines in Capella spectrum shown in Fig. 1

\begin{tabular}{|c|c|c|c|c|c|c|c|}
\hline$\lambda_{+1}^{a}$ & $I_{+1}^{b}$ & $\lambda_{-1}^{a}$ & $I_{-1}^{b}$ & $F^{c}$ & $\lambda_{\text {pred }}^{d}$ & $\operatorname{ion}^{d}$ & $\mathrm{ID}^{d}$ \\
\hline 6.20 & $15 \pm 3$ & 6.18 & $13 \pm 3$ & 0.7 & 6.180 & Si XIV & HLy $\alpha$ \\
\hline 6.66 & $43 \pm 5$ & 6.64 & $43 \pm 6$ & 2.0 & 6.648 & Si XIII & $\mathrm{Her}$ \\
\hline 6.70 & $13 \pm 4$ & 6.68 & $19 \pm 5$ & 0.7 & 6.688 & Si XIII & He $i$ \\
\hline 6.75 & $21 \pm 3$ & 6.73 & $22 \pm 3$ & 1.0 & 6.740 & Si XIII & $\operatorname{He} f$ \\
\hline 8.43 & $33 \pm 4$ & 8.41 & $36 \pm 4$ & 2.0 & 8.421 & Mg XII & HLy $\alpha$ \\
\hline 9.18 & $44 \pm 4$ & 9.16 & $50 \pm 5$ & 3.2 & 9.170 & Mg XI & $\mathrm{Her}$ \\
\hline 9.23 & $12 \pm 3$ & 9.22 & $9 \pm 3$ & 0.7 & 9.231 & Mg XI & $\mathrm{He} i$ \\
\hline 9.32 & $24 \pm 3$ & 9.31 & $23 \pm 4$ & 1.6 & 9.315 & Mg XI & $\mathrm{He} f$ \\
\hline 12.44 & $33 \pm 5$ & 12.42 & $25 \pm 5$ & 2.3 & 12.430 & Ni XIX & $\mathrm{Ne} 5$ \\
\hline 13.46 & $98 \pm 8$ & 13.45 & $89 \pm 7$ & 7.4 & 13.448 & Ne IX & $\mathrm{Her}$ \\
\hline 14.07 & $39 \pm 8$ & 14.06 & $40 \pm 8$ & 3.1 & 14.045 & Ni XIX & Ne8A \\
\hline 15.21 & $39 \pm 7$ & 15.20 & $40 \pm 8$ & 3.0 & 15.176 & O VIII & HLy $\gamma$ \\
\hline 18.64 & $20 \pm 4$ & 18.62 & $18 \pm 3$ & 1.7 & 18.627 & O VII & He3A \\
\hline 18.97 & $275 \pm 11$ & 18.96 & $309 \pm 11$ & 26 & 18.969 & O VIII & $\operatorname{HLy} \alpha$ \\
\hline 21.61 & $66 \pm 5$ & 21.61 & $71 \pm 5$ & 9.9 & 21.602 & O VII & $\mathrm{Her}$ \\
\hline 21.81 & $12 \pm 2$ & 21.81 & $15 \pm 2$ & 1.9 & 21.804 & O VII & He $i$ \\
\hline 22.11 & $46 \pm 4$ & 22.10 & $49 \pm 4$ & 6.9 & 22.101 & O VII & $\operatorname{He} f$ \\
\hline 24.79 & $46 \pm 5$ & 24.79 & $47 \pm 5$ & 6.9 & 24.781 & N VII & $\operatorname{HLy} \alpha$ \\
\hline 27.00 & $14 \pm 4$ & 27.01 & $17 \pm 4$ & 2.4 & 26.990 & C VI & HLy $\gamma$ \\
\hline 28.44 & $16 \pm 4$ & 28.43 & $14 \pm 3$ & 2.5 & 28.446 & C VI & HLy $\beta$ \\
\hline 28.79 & $10 \pm 3$ & 28.77 & $13 \pm 3$ & 1.9 & 28.787 & N VI & $\mathrm{Her}$ \\
\hline 29.10 & $5 \pm 2$ & 29.08 & $6 \pm 2$ & 0.9 & 29.084 & N VI & He $i$ \\
\hline 29.54 & $9 \pm 2$ & 29.52 & $11 \pm 2$ & 1.8 & 29.534 & N VI & $\operatorname{He} f$ \\
\hline 33.74 & $43 \pm 5$ & 33.74 & $41 \pm 4$ & 8.7 & 33.736 & C VI & HLy $\alpha$ \\
\hline 40.26 & $20 \pm 3$ & (sum & $+1 \&-1)$ & 5.0 & 40.268 & C V & $\mathrm{Her}$ \\
\hline 40.73 & $5 \pm 3$ & (sum & $+1 \&-1)$ & 1.9 & 40.731 & $\mathrm{C} \mathrm{V}$ & He $i$ \\
\hline 41.47 & $5 \pm 3$ & (sum & $+1 \&-1)$ & 2.2 & 41.472 & $\mathrm{C} \mathrm{V}$ & $\operatorname{He} f$ \\
\hline 43.78 & $22 \pm 3$ & 43.78 & $36 \pm 5$ & 3.2 & 43.763 & Si XI & Be5A \\
\hline 44.03 & $23 \pm 3$ & 44.02 & $29 \pm 4$ & 2.9 & 44.021 & Si XII & Li6A \\
\hline 44.17 & $41 \pm 5$ & 44.17 & $49 \pm 5$ & 5.1 & 44.165 & Si XII & Li6B \\
\hline 45.52 & $9 \pm 2$ & 45.51 & $11 \pm 2$ & 1.1 & 45.520 & Si XII & Li7B \\
\hline 45.69 & $16 \pm 3$ & 45.68 & $20 \pm 3$ & 2.1 & 45.692 & Si XII & Li7A \\
\hline 49.21 & $14 \pm 3$ & 49.20 & $14 \pm 7$ & 2.1 & 49.222 & Si XI & $\mathrm{Be} 8$ \\
\hline 57.89 & $18 \pm 2$ & 57.89 & $17 \pm 2$ & 3.4 & 57.876 & $\operatorname{Mg} X$ & Li5A \\
\hline gap & & 63.29 & $21 \pm 2$ & 2.4 & 63.295 & $\operatorname{Mg} X$ & Li6B \\
\hline
\end{tabular}

Notes to Tables 1 and 2

${ }^{a}$ observed wavelength in $\AA$.

${ }^{b}$ measured counting rate in $10^{-4} \mathrm{c} / \mathrm{s}$.

${ }^{c}$ line photon flux in $10^{-4} \mathrm{phot} / \mathrm{cm}^{2} / \mathrm{s}$, averaged over $+1 \&$ -1 order and corrected for interstellar absorption.

${ }^{d}$ predicted wavelength in $\AA$ and line identification (ID); see Phillips et al. (1999) (solar and laboratory measurements between 5 and $20 \AA$ ), Mason et al. (1984) (solar observations between 90 and $175 \AA$ ), and Mewe et al. (1985, 1995a) (full wavelength range in MEKAL code)

$e$ only total intensity is given because Fe XVIII and O VIII lines are blended

ASCA observations covering a broad wavelength range. Our work presented here covers the ASCA region and part of the EUVE region, though in more detail due to the higher spectral resolution $(\Delta \lambda \simeq 0.06 \AA)$ of the LETG
Table 2. Strongest iron lines in Capella spectrum shown in Fig. 1

\begin{tabular}{|c|c|c|c|c|c|c|}
\hline$\lambda_{+1}^{a}$ & $I_{+1}^{b}$ & $\lambda_{-1}^{a}$ & $I_{-1}^{b}$ & $F^{c}$ & $\lambda_{\text {pred }}^{d}$ ion $^{d}$ & $\mathrm{ID}^{d}$ \\
\hline 2.14 & $142 \pm 8$ & 12.13 & & 11 & $\begin{array}{l}\text { 12.124 Fe XVII } \\
12.134 \text { Ne IX }\end{array}$ & $\begin{array}{l}\mathrm{Ne} 4 \mathrm{C} \\
\mathrm{HLy} \alpha\end{array}$ \\
\hline 12.28 & $57 \pm 5$ & 12.27 & $61 \pm 6$ & 4.7 & 12.264 Fe XVII & $\mathrm{Ne} 4 \mathrm{D}$ \\
\hline 12.84 & $46 \pm 5$ & 12.83 & $49 \pm 6$ & 3.7 & $12.831 \mathrm{~F}$ & \\
\hline 13.83 & $90 \pm 7$ & 13.81 & $85 \pm 7$ & 6.8 & $13.826 \mathrm{Fe}$ & $\mathrm{Ne} 3 \mathrm{~A}$ \\
\hline 14.23 & $163 \pm 10$ & 14.22 & $186 \pm 10$ & 14 & 14.208 Fe XVIII & F14 \\
\hline 14.39 & $43 \pm 5$ & 14.38 & $39 \pm 4$ & 3.2 & $14.374 \mathrm{~F}$ & \\
\hline 14.56 & $36 \pm 5$ & 12.54 & $33 \pm 5$ & 2.7 & $14.540 \mathrm{l}$ & \\
\hline 15.03 & $426 \pm 1$ & 15.02 & & 34 & $15.014 \mathrm{I}$ & $\mathrm{Ne} 3 \mathrm{C}$ \\
\hline 15.28 & $145 \pm 9$ & 15.26 & $147 \pm 9$ & 11 & $15.265 \mathrm{I}$ & Ne3D \\
\hline 15.47 & $32 \pm 4$ & 15.46 & $37 \pm 4$ & 2.7 & $15.474 \mathrm{I}$ & $\mathrm{Ne} 3 \mathrm{E}$ \\
\hline 15.64 & $41 \pm 4$ & 15.62 & $53 \pm 6$ & 3.6 & & \\
\hline 15.84 & $35 \pm 5$ & 15.82 & $29 \pm 10$ & 2.5 & 15.831 & \\
\hline 15.88 & $37 \pm 5$ & 15.86 & $48 \pm 7$ & 3.3 & 15.873 & \\
\hline 16.02 & $107 \pm 7$ & 16.00 & $127 \pm 7$ & 9.1 & & \\
\hline & & & & & & \\
\hline 16.09 & $135 \pm 8$ & 16.07 & $151 \pm 10$ & 11 & & \\
\hline 16.78 & $259 \pm 1$ & 16.77 & $279 \pm$ & 20 & $16.780 \mathrm{H}$ & $\mathrm{Ne} 3 \mathrm{~F}$ \\
\hline 17.06 & $268 \pm 1$ & 17.05 & $446 \pm$ & 31 & $17.055 \mathrm{I}$ & Ne3G \\
\hline 17.10 & $309 \pm 16$ & 17.09 & $195 \pm$ & 24 & $17.100 \mathrm{~F}$ & $\mathrm{NeM}$ \\
\hline 17.63 & $38 \pm 4$ & 17.60 & $37 \pm 4$ & 3.6 & $17.626 \mathrm{~F}$ & \\
\hline & & & & & & \\
\hline 50.56 & $23 \pm 3$ & 50.56 & $11 \pm 3$ & 3.2 & & $\mathrm{Na} 6 \mathrm{~B}$ \\
\hline 52.90 & $4 \pm 2$ & gap & & 0.6 & $52.911 \mathrm{~F}$ & Mg1 \\
\hline 54.14 & $26 \pm 4$ & gap & & 3.9 & 54.1 & Na7B \\
\hline 54.72 & $37 \pm 4$ & gap & & 5.8 & 54 . & Na7A \\
\hline gap & & & $18 \pm 3$ & 2.0 & & $\mathrm{a} 8 \mathrm{~B}$ \\
\hline gap & & & & & & \\
\hline 66.40 & $16 \pm 3$ & 66.36 & $31 \pm 4$ & 3.3 & $66.368 \mathrm{~F}$ & \\
\hline 91.06 & $14 \pm 3$ & 91.01 & $15 \pm 3$ & 3.2 & $91.020 \mathrm{~F}$ & $\mathrm{O} 6 \mathrm{~F}$ \\
\hline 93.95 & $160 \pm 8$ & 93.92 & $159 \pm 8$ & 37 & $93.923 \mathrm{~F}$ & $\mathrm{~F} 4 \mathrm{~A}$ \\
\hline 97.90 & 5 & & & 0.9 & 97.0 & $6 \mathrm{C}$ \\
\hline 01.58 & $28 \pm 3$ & 101.55 & $27 \pm 3$ & 7.6 & $101.550 \mathrm{Fe}$ & O6B \\
\hline 02.26 & $10 \pm 2$ & 102.22 & $9 \pm 2$ & 2.6 & $102.220 \mathrm{Fe}$ & \\
\hline 103.97 & $54 \pm 5$ & 103.95 & $54 \pm 5$ & 15 & $103.937 \mathrm{~F}$ & \\
\hline 108.38 & $87 \pm 6$ & 108.36 & $94 \pm 6$ & 25 & 108.370 Fe XIX & O6A \\
\hline 109.98 & $12 \pm 2$ & 109.98 & & 2.9 & $109.970 \mathrm{~F}$ & $\mathrm{O} 6 \mathrm{E}$ \\
\hline 10.67 & $3 \pm 1$ & 110.64 & $4 \pm 1$ & 0.9 & $110.630 \mathrm{Fe}$ & N6D \\
\hline 111.72 & $9 \pm 3$ & 11.70 & $7 \pm 1$ & 2.2 & $111.700 \mathrm{Fe}$ & O6 \\
\hline 114.41 & & & $4 \pm 1$ & 1.1 & 114.410 & \\
\hline 117.14 & $24 \pm 3$ & 117.16 & $22 \pm 3$ & 6.4 & 117.170 Fe XXII & B11 \\
\hline 117.50 & $8 \pm 3$ & 117.53 & $4 \pm 2$ & 1.7 & 117.510 Fe XXI & C7B \\
\hline 118.70 & $17 \pm 3$ & 118.70 & $23 \pm 3$ & 5.7 & $118.660 \mathrm{Fe} \mathrm{X}$ & $\mathrm{N} 6 \mathrm{C}$ \\
\hline 20.00 & $22 \pm 3$ & 120.01 & $24 \pm 4$ & 6.7 & 120.000 Fe XIX & O6D \\
\hline 21.87 & $36 \pm 4$ & 121.88 & $33 \pm 4$ & 11 & $121.830 \mathrm{Fe} \mathrm{XX}$ & N6B \\
\hline 128.76 & $22 \pm 3$ & 128.74 & $22 \pm 3$ & 11 & 128.730 Fe XXI & C6A \\
\hline 132.87 & $37 \pm 4$ & 132.87 & $51 \pm 5$ & 20 & 132.850 Fe XX & \\
\hline 135.81 & $11 \pm 3$ & 135.82 & $12 \pm 3$ & 5.4 & 135.780 Fe XXII & $\mathrm{B} 12 \mathrm{~A}$ \\
\hline 171.06 & $39 \pm 5$ & off & & 44 & 171.075 Fe IX & $\mathrm{A} 4$ \\
\hline 174.51 & $6 \pm 2$ & off & detector & 18 & $174.535 \mathrm{Fe} \mathrm{X}$ & $\mathrm{Cl} 4 \mathrm{~A}$ \\
\hline
\end{tabular}


spectrometer. In the present paper, the long-wavelength iron lines have been studied for the purposes of providing further insights into the coronal temperature structure as well as for density diagnostics. Section 2 discusses the observations, the data reduction, the counting rate/photon flux conversion, and the correction for interstellar absorption. Section 3 discusses branching ratios for $n_{\mathrm{e}}$-independent lines decaying from the same upper level or within the same ion in relation to theoretical values and the consequences for optical depth and source size. In Sect. 4 the analysis of the temperature diagnostic for helium-like triplets and iron lines is presented and Sect. 5 discusses briefly the determination of the electron density from the iron lines and the helium-like triplets. Section 6 describes the emission measure distribution and Sect. 7 discusses the results in terms of quasi-static loop models.

\section{Observations}

Spectra of Capella were obtained in the calibration phase during 6-12 September 1999 (cf. Brinkman et al. 2000) and subsequently on 9 November 1999, using the High Resolution Camera (HRC-S) and the Low Energy Transmission Grating (LETG) on board the X-ray observatory CHANDRA. In the present paper we study the second spectrum (exposure time $85.4 \mathrm{ksec}$ ).

The HRC-S is equipped with three microchannel plate detectors $(10 \mathrm{~cm}$ each) in one row. The 0th order diffraction signal is aimed at the central detector, such, that for the +1 and -1 diffraction orders, the wavelength regions that fall in the gaps between the detector plates do not overlap (see Fig. 1). This way the central plate covers nominally the range of $-50(-1$ order $)$ to $+60(+1$ order $)$ $\AA$, the outer plates -55 to $-165 \AA$ and +65 to $+175 \AA$. The dispersion is $1.15 \AA / \mathrm{mm}$. Figure 1 shows the LETGS spectra of Capella in the $+1 \&-1$ order with the gaps between $62-65 \AA$ and $52-56 \AA$ ( +1 and -1 order, respectively) and a detail of the -1 order spectrum between 12-25 $\AA$. The spectra were rebinned to intervals of $0.02 \AA$ and corrected for the background. Around the dispersion axis a source box is defined. The edge of the box is a function of the wavelength, small at short wavelengths and wide at long wavelengths, like a bowtie. The background box is defined as the area from 10 to 39.5 arcsec above and under the dispersion axis. The ratio of the source to background area ranges from 1:25 at the short wavelengths to 1:6 at the edge of the detector. For each bin the number of counts is integrated in the cross dispersion direction inside the boxes. The net number of source counts is calculated as follows: $s=$ raw $-f \cdot$ back, $\mathrm{d} s=\sqrt{\text { raw }+f^{2} \cdot \text { back }}, f=\frac{\text { area(source) }}{\text { area(back) }}$, where back $=\#$ counts in background box, raw = \# counts in source box, and $s=\#$ net source counts in source box with error $\mathrm{d} s$.

By visual inspection we selected the prominent lines and fitted them with Gaussian profiles. This has yielded values for the wavelength, FWHM, and the number of photon counts (including uncertainties) for each line. By comparing wavelengths and line counts with expected val- ues we have identified all lines given in Tables 1 and 2. To represent the background due to continuum radiation we introduce a constant shift. The intensities of the emission line features in the spectrum are based on integration over the line profile to obtain the total counts. For isolated lines the Gaussian line width turns out to be consistent with the average theoretical instrumental width, which implies that Doppler broadening can be neglected compared to the instrumental width. The measured FWHM of the instrumental profile is about $0.068 \AA$ for wavelengths $\lambda \lesssim 50 \AA$ (and increasing with $\lambda$ at long wavelengths). This is in good agreement with the results of the pre-flight ground calibration. Blended lines were fitted by multiple Gauss profiles with the proper instrumental width. The LETGS instrumental profile is actually closer to a Lorentzian function than to a Gaussian; we have investigated the effect of using Lorentzian profiles in the analysis but usually found no significant differences in derived line parameters.

Counting rates $I$ and $1 \sigma$ uncertainties are given in Tables 1 and 2 . The observed counting rates $I$ $\left(10^{-4} \mathrm{c} / \mathrm{s}\right)$ have been converted to photon fluxes $F$ $\left(10^{-4} \mathrm{phot} / \mathrm{cm}^{2} / \mathrm{s}\right)$ using the preliminary in-flight effective area model for the spectrometer (for details of the calibration, see van der Meer et al. 2001). The observed flux at Earth was further corrected for interstellar absorption, assuming an interstellar column density $N_{\mathrm{H}}=1.810^{18} \mathrm{~cm}^{-2}$ (see Schrijver et al. 1995), using the cross sections of Morrison \& McCammon (1983). In Tables 1 and 2 we give the photon flux $F$ averaged over the $+1 \&-1$ order and corrected for interstellar absorption.

In the overlapping wavelength regions $(\lambda<35 \AA)$ the obtained fluxes are in agreement with values obtained with the CHANDRA-HETGS by Canizares et al. (2000) and with data from the RGS on board XMM-Newton (see Audard et al. 2001; cf. their Table 1 for a comparison of fluxes). For the long-wavelength region $(\lambda>90 \AA)$ we have compared the LETGS fluxes with the EUVE fluxes obtained by Brickhouse et al. (2000). The LETGS/EUVE flux ratio was in the range $\sim 0.5$ to 0.8 which is explained by a different activity level of Capella (see e.g., comparison by Linsky et al. 1998).

\section{Comparison of observations with models}

This section presents a comparison of observations with model predictions. First, we consider branching ratios for lines decaying from the same upper level and some optical depth effects. Second, we consider some lines decaying from different upper levels.

\subsection{Branching ratios}

The branching ratio is defined as the ratio of the photon fluxes of two lines decaying from the same upper level. The uncertainty of the branching ratio is given by the uncertainty of the radiative transition probabilities $\mathrm{A}$ of the lines which is typically about $25 \%$ each (see below). The comparison of the measured and theoretical branching 
Table 3. Comparison of branching ratios

\begin{tabular}{l|ccccccc}
$\lambda(\AA)$ & $+1^{a}$ & $-1^{a}$ & av. $^{b}$ & $N^{c}$ & $M^{d}$ & $C^{e}$ & $B^{f}$ \\
\hline $\begin{array}{l}\text { Si XII } \\
\frac{45.52}{45.692}\end{array}$ & 0.57 & 0.52 & $0.54(.15)$ & 0.51 & 0.50 & 0.51 & - \\
Fe XVI & & & & & & & \\
$\frac{62.87}{63.719}$ & gap & 0.50 & $0.49(.08)$ & 0.48 & 0.48 & 0.52 & - \\
Fe XVIII & & & & & & & \\
$\frac{93.92}{103.94}$ & 3.0 & 2.9 & $2.5(.2)$ & 2.76 & 2.43 & 2.70 & 2.71 \\
Fe XIX & & & & & & & \\
$\frac{108.37}{120.0}$ & 4.0 & 4.0 & $3.5(.4)$ & 3.75 & 3.51 & 3.64 & 3.75 \\
$\frac{101.55}{109.97}$ & 2.3 & 3.0 & $2.6(.4)$ & 1.98 & 1.80 & 1.95 & 2.00 \\
$\frac{101.55}{111.7}$ & 3.3 & 3.8 & $3.5(1.1)$ & 2.52 & 2.34 & 2.51 & 2.54 \\
$\frac{109.97}{111.7}$ & 1.42 & 1.28 & $1.4(.4)$ & 1.27 & 1.27 & 1.29 & 1.27 \\
Fe XXI & & & & & & & \\
$\frac{102.22}{97.88}$ & 2.0 & 4.0 & $2.8(1.7)$ & 2.42 & 2.37 & 2.39 & 2.42 \\
\hline
\end{tabular}

${ }^{a}$ measured counting rate ratio in +1 and -1 order of spectrum.

${ }^{b}$ line photon flux ratio averaged over +1 and -1 order and corrected for interstellar absorption (in parentheses the mean statistical $1 \sigma$ error).

${ }^{c}$ from ratio of transition probabilities $A$ given by NIST database with uncertainty classification $\mathrm{C}(<25 \%)$.

${ }^{d}$ calculated with SPEX/MEKAL code (Mewe et al. 1985, 1995a; Kaastra et al. 1996).

${ }^{e}$ from recent calculations of transition probabilities by Raassen using Cowan (1981) code.

${ }^{f}$ from Brickhouse et al. (1995).

ratios provides a good figure of merit for the quality of the flux measurements as well as a check on the assumption of the optically thin model, i.e., optical line depth $\tau \simeq 0$.

In Table 3, photon flux ratios of two lines decaying from the same upper level are compared to the theoretical branching ratios. The theoretical values are from the NIST compilation, from MEKAL (Mewe et al. 1985, 1995a) as implemented in the SPEX code (Kaastra et al. 1996), from recent calculations at SRON using the Cowan code programs, and from work by Brickhouse et al. (1995). From this table we notice that the theoretical ratios from MEKAL for the Fe XVIII pair and three of the Fe XIX pairs are somewhat lower than those from the three other theories, which are in good agreement with each other. For all except one the theoretical line ratios in Table 3 are within the experimental statistical errors of the Capella line ratios. For some of the ratios the statistical error is also very large, e.g., the ratio $102.22 / 97.88$ for which the deviation between the +1 and -1 order is apparently large, though the flux for $97.88 \AA$ should only be treated as an upper limit. The only disagreement concerns the ratio 101.55/109.97. However, taking into account an uncertainty of $25 \%$ for the theoretical values as indicated by the NIST database (classification " $C$ ") all ratios agree with the theoretical values. Brickhouse et al. (1995) observed a deviation for the ratio $101.55 / 111.7$. She suggests a blend for the line at $101.55 \AA$. Although in our case the ratios related to the $101.55 \AA$ line are about $25 \%$ larger than the theoretical value (which is in agreement with some blending) they are all within the combined statistical and NIST error bars.

\subsubsection{Optical depth effects}

Schrijver et al. (1995) and Mewe et al. (1995b) have investigated the possibility that strong resonance photons are scattered out of the line of sight in late-type stellar coronae. Branching ratios can be used to check the assumption of the optical thin model because effects of resonance scattering would affect the measured branching ratio. From the fact that the intensities of, e.g., the strong resonance lines Fe XVIII $\lambda 93.92$ and Fe XIX $\lambda 108.37$ are in good agreement with the intensities of other lines sharing the same upper levels, we can derive a constraint on the optical depth taking into account the systematic uncertainties of the theoretical transition probabilities A (typical $25 \%$ for each $\mathrm{A}$, hence $35 \%$ for the branching ratio) which dominate over the statistical errors (typically 10\%). If we rule out a reduction in the resonance line intensity larger than about $30 \%$, then on the basis of a simple "escapefactor" model (escape factor $P(\tau) \simeq[1+0.43 \tau]^{-1}$ for a homogeneous mixture of emitters and absorbers in a slab geometry (e.g., Kaastra \& Mewe 1995)) we can put a constraint on the optical depth: $P \gtrsim 0.7$ yields $\tau \lesssim 1$ for the Fe XVIII $\lambda$ 93.92 line.

Line transitions of Ne-like Fe XVII yield the strongest emission lines seen in the LETGS spectrum in the range 15-17 $\AA$ (cf. Fig. 1 and Table 2). For the very strong Fe XVII $\lambda 15.014$ resonance line we can also derive a constraint on the optical depth as follows.

In principle, the ratio of the strong Fe XVII $15.014 \AA$ resonance line (with a large oscillator strength $f=2.66$ ) to a presumably optically thin Fe XVII line with a relatively small oscillator strength (e.g., $16.780 \AA, f=0.101$, or $15.265 \AA, f=0.593)$ yields the optical depth $\tau$ in the $15.014 \AA$ line, applying the escape-factor model. Solar physicists have used this technique to derive the density in active regions on the Sun from the optical depth and the estimated plasma dimension $\ell$ (e.g., Saba et al. 1999). Though the $16.780 \AA$ line has the smallest $f$-value we prefer to use the $15.265 \AA$ line because the $16.780 \AA 3 \mathrm{~s} \rightarrow 2 \mathrm{p}$ line can be affected by radiative cascades.

From the measured Fe XVII 15.014/15.265 photon flux ratio of $2.85 \pm 0.14$ we derive for a plasma of $7 \mathrm{MK}$ (as derived from the Fe XVII 15.265/Fe XVIII 16.078 ratio, see Sect. 4.1) an upper limit to the optical depth $\tau$ of the Fe XVII 15.014 $\AA$ line by comparing to the corresponding line ratios given by experiment (2.8-3.2) (Livermore Electron Beam Ion Trap (EBIT)) or theory (3.3-4.7) (cf. Brown et al. 1998; Bhatia \& Doschek 1992). Taking the EBIT ratio we obtain from the condition $P \gtrsim 2.85 / 3=$ 0.95 an upper limit of $\tau \lesssim 0.3$, whereas the theoretical 
ratio would give a more conservative upper limit of $\tau \lesssim 1.5$ from $P \gtrsim 2.85 / 4.7=0.61$.

\subsubsection{Constraints on the source size}

The constraints on $\tau$ imply a constraint on the extent of the emitting plasma though the averaging over the stellar surface makes a straightforward interpretation difficult. The optical depth $\tau$ for a Doppler-broadened resonance line can be written as (Mewe et al. 1995b):

$$
\begin{aligned}
\tau & =1.1610^{-14}\left(\frac{n_{\mathrm{i}}}{n_{\mathrm{el}}}\right) A_{z}\left(\frac{n_{\mathrm{H}}}{n_{\mathrm{e}}}\right) \lambda f \sqrt{\frac{M}{T}} n_{\mathrm{e}} \ell \\
& \equiv 10^{-19} C_{\mathrm{d}}\left(\frac{A_{z}}{A_{z \odot}}\right)\left(\frac{n_{\mathrm{e}} \ell}{\sqrt{T_{6}}}\right),
\end{aligned}
$$

where $\left(n_{\mathrm{i}} / n_{\mathrm{el}}\right)$ is the ion fraction (from Arnaud \& Raymond 1992), $A_{z}=n_{\mathrm{el}} / n_{\mathrm{H}}$ the elemental abundance relative to hydrogen with $A_{z} \odot$ the corresponding value for the solar photosphere as given by Anders \& Grevesse (1989), $n_{\mathrm{H}} / n_{\mathrm{e}} \simeq 0.85$ the ratio of hydrogen to electron density (in $\mathrm{cm}^{-3}$ ), $\lambda$ the wavelength in $\AA, f$ the absorption oscillator strength, $M$ the atomic weight, $T$ the temperature (in $\mathrm{K}$ or $T_{6}$ in $\mathrm{MK}$ ), and $\ell$ a characteristic plasma dimension (in $\mathrm{cm}$ ), and

$C_{\mathrm{d}} \equiv 98.5\left(n_{\mathrm{i}} / n_{\mathrm{el}}\right) A_{z \odot} \lambda f \sqrt{M}$.

For Fe XVII $\lambda 15.014$, Fe XVIII $\lambda 93.92$, and Fe XIX $\lambda 108.37$ we obtain from Eq. (2) $C_{\mathrm{d}} \simeq 0.25,0.05$, and 0.04 , respectively for a temperature $T_{6} \simeq 6.5$ (see Sect. 4). Assuming solar abundances $\left(A_{z}=A_{z \odot}=4.6810^{-5}\right.$ for $\mathrm{Fe}$ ), the constraints on $\tau$ yield corresponding upper limits for $n_{\mathrm{e}} \ell$ of about $210^{20}, 510^{20}$, and $610^{20} \mathrm{~cm}^{-2}$ for these three cases. With a density of typically $n_{\mathrm{e}} \approx 310^{12} \mathrm{~cm}^{-3}$ (see Sect. 5.1) we derive upper limits to the typical size $\ell$ of the emitting region in the range $(1-2) 10^{8} \mathrm{~cm}$, i.e. much smaller than the stellar radii $\left(\sim 10 R_{\odot} \simeq 10^{12} \mathrm{~cm}\right)$, but slightly above the typical loop size as derived from the electron density in a comparable temperature region (cf. Table 9, Fe XX-XXI). For the Sun loop sizes typically range from $310^{9} \mathrm{~cm}$ for compact active region loops to $210^{10} \mathrm{~cm}$ for large-scale quiet region loops (see e.g., Mewe 1991, Table 1).

The above analysis, however, is strictly valid only if all flux is concentrated in one single loop. If one observes a collection of loops distributed more or less homogeneously over the stellar surface, the effects of optical depth tend to average out. There may be significant resonance scattering of photons into the line of sight. In this situation it is possible that there is indeed significant optical depth in certain structures, but that this is compensated for by the azimuthal average over the stellar disk whereby some lines of sight pick up photons that are scattered and others lose photons to scattering (e.g., Wood \& Raymond 2000). Nevertheless, the above analysis still serves to demonstrate the usefulness of investigating optical depths to provide further clues on the emitting geometry.
Table 4. Line ratios based on different upper levels

\begin{tabular}{l|ccccc}
$\lambda(\AA)$ & $+1^{a}$ & $-1^{a}$ & av. $^{b}$ & $M^{c}$ & $B^{d}$ \\
\hline Fe XVI & & & & & \\
$54.14 / 54.728$ & 0.70 & gap & $0.67(.15)$ & 0.56 & - \\
& & & & & \\
Fe XIX & & & & & \\
$91.02 / 101.55$ & 0.49 & 0.56 & $0.42(.07)$ & 0.156 & 0.241 \\
$91.02 / 108.37$ & 0.16 & 0.16 & $0.130(.014)$ & 0.057 & 0.082 \\
$91.02 / 109.97$ & 1.1 & 1.7 & $1.09(.19)$ & 0.287 & 0.476 \\
$108.37 / 109.97$ & 7.0 & 10.4 & $8.4(1.4)$ & 5.00 & 5.48 \\
$108.37 / 111.70$ & 10.0 & 13.6 & $11.4(2.6)$ & 6.34 & 6.95 \\
& & & & & \\
Fe XX & & & & & \\
$118.66 / 121.83$ & 0.48 & 0.68 & $0.55(.07)$ & 0.517 & 0.517 \\
$118.66 / 132.85$ & 0.47 & 0.44 & $0.28(.03)$ & 0.355 & 0.386 \\
$121.83 / 132.85$ & 0.97 & 0.65 & $0.55(.06)$ & 0.679 & 0.754 \\
& & & & & \\
Fe XXII & & & & & \\
$117.17 / 135.78$ & 2.1 & 1.8 & $1.17(.2)$ & 0.853 & 0.991 \\
\hline
\end{tabular}

${ }^{a}$ measured counting rate ratio in +1 and -1 order of spectrum.

${ }^{b}$ line photon flux ratio averaged over +1 and -1 order and corrected for interstellar absorption (in parentheses the mean statistical $1 \sigma$ error).

${ }^{c}$ calculated with SPEX/MEKAL code (Mewe et al. 1985, 1995a; Kaastra et al. 1996).

${ }^{d}$ Brickhouse et al. (1995).

\subsection{Lines decaying from different upper levels}

In Table 4 ratios are given for lines that belong to the same Fe ion, but that do not decay from the same upper level. In this case they cannot be compared to the ratios of the transition probabilities, the branching ratio as given by NIST and COWAN. However, comparison with MEKAL and Brickhouse et al. (1995) is still possible, as they take into account the populations of the levels due to collisional excitations and radiative decays. Only densityindependent lines are used. In Table 4 all ratios in Fe XIX that include the line at $91.02 \AA$ are far too high. This implies blending by an unknown feature or a theoretical flux value far too low for that line. There is some indication for this, given the considerable difference between the MEKAL and Brickhouse values, suggesting some uncertainties in the calculation of the proper flux value. This situation renders this line at $91.02 \AA$ problematic for density and temperature diagnostics. The ratios including the Fe XX line at $132.85 \AA$ also deviate from the theoretical values. However, $132.85 \AA$ is well known to be blended with a line of Fe XXIII; the relative contributions of Fe XX and Fe XXIII depend on the temperature regime and for Capella are expected to be dominated by Fe XX. The deviant behaviour of the lines at $91.02 \AA$ and $132.85 \AA$ appears again in Sects. 4 and 5 where we discuss the temperature and density diagnostics. For completeness, these lines were included in the tables though the results based on their ratios should be considered as less reliable. 

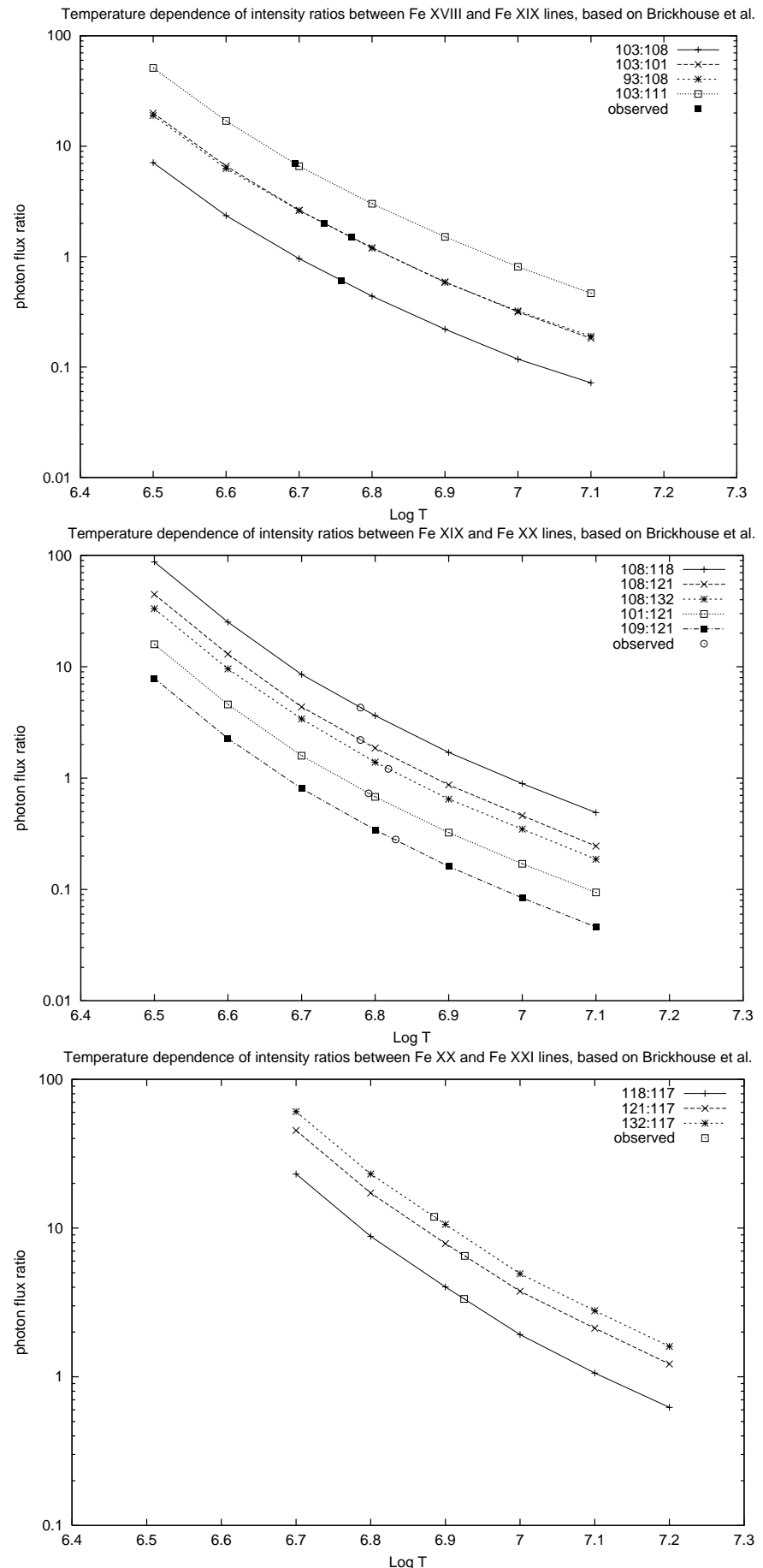

Fig. 2. Temperature dependence of photon flux ratios between Fe XVIII to Fe XXI lines. The observed ratios are indicated

\section{Temperature diagnostics}

\subsection{Fe ions}

In Fig. 3 and Table 5 theoretical ratios of temperaturesensitive lines of higher charge state ions of iron are shown and compared with the observations (Brickhouse et al. 1995). The line ratios from the lower charge state Fe ions Fe IX and Fe $\mathrm{X}$ indicate that these ions are formed in a plasma with a temperature of about $1 \mathrm{MK}$. For the higher Fe ions Fe XVIII, Fe XIX, and Fe XX temperatures of
Table 5. Temperature determination from line ratios for different Fe ions

\begin{tabular}{|c|c|c|c|c|}
\hline$\lambda(\AA)$ & $+1^{a}$ & $-1^{a}$ & av. $^{b}$ & $T^{c}$ \\
\hline Fe IX/Fe X & & & & \\
\hline $171.07 / 174.53$ & 6.36 & - & $\sim 2.4(.8)$ & $\sim 0.85$ \\
\hline \multicolumn{5}{|l|}{ Fe XVII/Fe XVIII } \\
\hline $15.265 / 16.078$ & 1.07 & 0.97 & $1.01(.07)$ & $7.0 \pm 0.2$ \\
\hline $16.780 / 15.628$ & 6.36 & 5.30 & $5.7(.6)$ & $6.9 \pm 0.2$ \\
\hline \multicolumn{5}{|l|}{ Fe XVIII/Fe XVIII } \\
\hline $15.628 / 93.92$ & 0.25 & 0.33 & $0.10(.01)$ & $10.0 \pm 1.0$ \\
\hline \multicolumn{5}{|l|}{ Fe XVIII/Fe XIX } \\
\hline $103.94 / 108.37$ & 0.63 & 0.58 & $0.61(.05)$ & $5.7 \pm 0.2$ \\
\hline $103.94 / 101.55$ & 1.9 & 2.0 & $2.0(.2)$ & $5.4 \pm 0.2$ \\
\hline $93.92 / 91.02^{d}$ & 11.5 & 10.6 & $11.6(1.6)$ & $6.6 \pm 0.3$ \\
\hline $93.92 / 108.37$ & 1.84 & 1.70 & $1.51(.15)$ & $5.9 \pm 0.2$ \\
\hline $103.94 / 111.70$ & 6.26 & 7.72 & $7.0(1.5)$ & $5.0 \pm 0.4$ \\
\hline \multicolumn{5}{|l|}{ Fe XIX/Fe XX } \\
\hline $120.00 / 118.66$ & 1.3 & 1.0 & $1.2(.2)$ & $6.0 \pm 0.3$ \\
\hline $120.00 / 121.83$ & 0.61 & 0.70 & $0.63(.07)$ & $5.9 \pm 0.2$ \\
\hline $120.00 / 132.85^{d}$ & 0.59 & 0.46 & $0.35(.04)$ & $6.3 \pm 0.3$ \\
\hline $108.37 / 118.66$ & 5.0 & 4.1 & $4.3(.5)$ & $6.0 \pm 0.3$ \\
\hline $108.37 / 121.83$ & 2.4 & 2.8 & $2.2(.2)$ & $6.0 \pm 0.2$ \\
\hline $108.37 / 132.85^{d}$ & 2.4 & 1.8 & $1.21(.09)$ & $6.6 \pm 0.2$ \\
\hline $111.70 / 118.66$ & 0.50 & 0.31 & $0.38(.12)$ & $7.0_{-0.6}^{+0.8}$ \\
\hline $111.70 / 121.83$ & 0.24 & 0.21 & $0.21(.06)$ & $6.8_{-0.5}^{+0.9}$ \\
\hline $101.55 / 121.83$ & 0.79 & 0.79 & $0.73(.07)$ & $6.2 \pm 0.3$ \\
\hline $109.97 / 121.83$ & 0.35 & 0.27 & $0.28(.05)$ & $6.7 \pm 0.5$ \\
\hline $91.02^{d} / 121.83$ & 0.39 & 0.45 & $0.31(.03)$ & $5.3 \pm 0.2$ \\
\hline \multicolumn{5}{|l|}{ Fe XX/Fe XXI } \\
\hline $118.66 / 117.51$ & 2.2 & 5.2 & $3.3(1.0)$ & $8.4_{-0.7}^{+1.0}$ \\
\hline $121.83 / 117.51$ & 4.6 & 7.6 & $6.5(2.0)$ & $8.4_{-0.7}^{+0.9}$ \\
\hline $132.85^{d} / 117.51$ & 4.8 & 11.7 & $11.9(3.9)$ & $\begin{array}{l}-0.7 \\
7.7_{-0.5}^{+0.9}\end{array}$ \\
\hline \multicolumn{5}{|l|}{ Fe XXI/Fe XXII } \\
\hline $117.51 / 117.17$ & 0.32 & 0.20 & $0.27(.08)$ & $9.7_{-0.8}^{+1.2}$ \\
\hline
\end{tabular}

${ }^{a}$ measured counting rate ratio in +1 and -1 order of spectrum.

${ }^{b}$ line photon flux ratio averaged over +1 and -1 order and corrected for interstellar absorption (in parentheses the mean statistical $1 \sigma$ error).

${ }^{c}$ Temperature (in MK) derived from Brickhouse et al. (1995) but for the 15-16 $\AA$ Fe XVII/Fe XVIII line ratios from corrected MEKAL (see text).

${ }^{d}$ line intensity too high compared to theoretical values (see Table 4 and paragraph 3.2); results less reliable.

$6 \mathrm{MK}$ or more are observed, but for ions higher than Fe XX the temperature increases to 8 or $10 \mathrm{MK}$. These results are consistent with those obtained by Brickhouse et al. (2000) on the basis of EUVE observations.

We notice some deviations including the Fe XIX lines at 108 and $120 \AA$ and those at 101, 109, and $111 \AA$. The first two both decay from the same upper level 
Table 6. Temperature determination from the line intensity ratio $G=(i+f) / r$ for different He-like ions and from the ratio of the He- and H-like resonance lines.

\begin{tabular}{|c|c|c|c|c|c|}
\hline$\lambda(\AA)$ & $+1^{a}$ & $-1^{a}$ & av. ${ }^{b}$ & $T^{c}$ & $T^{d}$ \\
\hline $\mathrm{C} \mathrm{V}$ & & & & & \\
\hline$\frac{40.731+41.472}{40.268}$ & sum & & $0.80(.26)$ & $1.4 \pm 0.5$ & 1.0 \\
\hline N VI & & & & & \\
\hline$\frac{29.084+29.534}{28.787}$ & 1.44 & 1.28 & $1.44(.46)$ & $0.5_{-0.2}^{+0.5}$ & 0.35 \\
\hline O VII & & & & & \\
\hline$\frac{21.804+22.101}{21.602}$ & 0.88 & 0.90 & $0.89(.09)$ & $1.8 \pm 0.3$ & 1.9 \\
\hline $\begin{array}{l}\mathrm{Mg} \mathrm{XI} \\
\frac{9.231+9.315}{9.170}\end{array}$ & 0.83 & 0.67 & $0.74(.11)$ & $4.6_{-1.0}^{+1.4}$ & 5.7 \\
\hline $\begin{array}{l}\text { Si XIII } \\
\frac{6.688+6.740}{6.648}\end{array}$ & 0.78 & 0.94 & $0.87(.17)$ & $5_{-2}^{+3}$ & 6 \\
\hline $\begin{array}{l}\text { C V/C /C VI } \\
\frac{40.268}{33.736}\end{array}$ & sum & & $0.59(.24)$ & $1.10 \pm 0.15$ & \\
\hline $\begin{array}{l}\text { N VI/VII } \\
\frac{28.787}{24.781}\end{array}$ & 0.213 & 0.269 & $0.27(.04)$ & $2.50 \pm 0.14$ & \\
\hline $\begin{array}{l}\text { O VII/VIII } \\
\frac{21.602}{18.969}\end{array}$ & 0.239 & 0.229 & $0.37(.02)$ & $3.37 \pm 0.06$ & \\
\hline $\begin{array}{l}\mathrm{Mg} \text { XI/XII } \\
\frac{9.170}{8.421}\end{array}$ & 1.34 & 1.38 & $1.61(.18)$ & $6.9 \pm 0.2$ & \\
\hline $\begin{array}{l}\text { Si XIII/XIV } \\
\frac{6.648}{6.182}\end{array}$ & 2.79 & 3.33 & $2.82(.44)$ & $9.4 \pm 0.5$ & \\
\hline
\end{tabular}

${ }^{a}$ measured counting rate ratio in +1 and -1 order of spectrum.

${ }^{b}$ line photon flux ratio averaged over +1 and -1 order and corrected for interstellar absorption (in parentheses the mean statistical $1 \sigma$ error).

${ }^{c}$ Temperature (in MK) derived from SPEX/MEKAL.

${ }^{d}$ Temperature (in MK) derived from Porquet \& Dubau (2000) and Mewe et al. (2001).

$\left(922.908 \mathrm{~cm}^{-1}\right)$, while the three others all decay from the level at $984.760 \mathrm{~cm}^{-1}$. In the ratio between the Fe XIX/Fe XX this results in a lower temperature when the two lines at 108 and $120 \AA$ are involved and a somewhat higher temperature when the three other lines are used. Errors induced in the computed populations of the levels in the theoretical model resulting from inaccurate collisional excitation rates are likely to be the underlying reason. We notice from Table 5 a fairly regular increase of the temperature from $6 \mathrm{MK}$ up to $10 \mathrm{MK}$ going from Fe XVIII to Fe XXII. This favours a multi-temperature model; this comes as no surprise based on the earlier analyses using EUVE and ASCA observations that arrived at similar conclusions (e.g., Dupree et al. 1993; Brickhouse et al. 2000).

The wide dynamic wavelength range of the LETGS allows to study simultaneously the $n=2 \rightarrow 2$ and $n=$ $3 \rightarrow 2$ iron line transitions in the L-shell and to compare the derived temperatures. In the short wavelength range, around $16 \AA$ the observed features correspond to $n=3 \rightarrow$ 2 transitions, while in the wavelength range above $80 \AA$ the lines originate from $n=2 \rightarrow 2$ transitions. From the

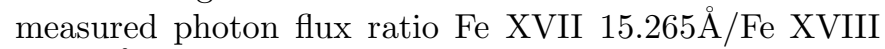
$16.078 \AA=1.01 \pm 0.07$ we derive a temperature of $7.0 \pm$ $0.2 \mathrm{MK}$ using MEKAL, but after correcting (enhancing) the Fe XVIII flux by a factor 2.14 as indicated by the results from a benchmark study of the MEKAL code with solar flare spectra by Phillips et al. (1999; their Table A8). The two Fe XVII/Fe XVIII $n=3 \rightarrow 2$ ratios result in higher temperatures than the ratios based on $n=2 \rightarrow$ 2 transitions. As a test case the ratio Fe XVIII/Fe XVIII between an $n=3 \rightarrow 2$ and an $n=2 \rightarrow 2$ transition was calculated. It results in an even higher temperature of $10 \mathrm{MK}$. However, this ratio covers a large wavelength range and is more sensitive to flux calibration errors.

Higher temperature regions $(T=10 \mathrm{MK})$ are present in the coronae of Capella (e.g., Brickhouse et al. 2000), though with emission measures more than an order of magnitude below those of the dominant temperature of several MK. Lines of higher Fe ionization stages (e.g., Fe XXIV) that could constrain higher temperatures are outside the range in which the LETGS has either insufficient spectral resolution or sensitivity to yield accurate measurents of their fluxes.

\subsection{He-like ions}

The LETGS spectra of Capella contain a number of interesting emission line features in the short wavelength region between 6 and $45 \AA$ that originate from the Helike ions Si XIII, Mg XI, Ne IX, O VII, N VI, and C V. It concerns the resonance line $1 \mathrm{~s}^{2}{ }^{1} \mathrm{~S}_{0}-1 \mathrm{~s} 2 \mathrm{p}{ }^{1} \mathrm{P}_{1}(r)$, the intercombination line $1 \mathrm{~s}^{2}{ }^{1} \mathrm{~S}_{0}-1 \mathrm{~s} 2 \mathrm{p}{ }^{3} \mathrm{P}_{1,2}(i)$ and the forbidden line $1 \mathrm{~s}^{2}{ }^{1} \mathrm{~S}_{0}-1 \mathrm{~s} 2 \mathrm{~s}{ }^{3} \mathrm{~S}_{1}(f)$. There are combinations of these three transitions that are strongly temperatureand density-sensitive. The resonance line intensity is comparable to the sum of the intensities of the two other lines and increases at higher temperatures, while for high density the intercombination line becomes intense compared to the forbidden line. Of the six ions mentioned above, the Ne IX triplet could not be used because of severe blending of the $r$ and $i$ lines by Fe XIX lines and partly blending of the $f$ line by an Fe XVII line. For $\mathrm{C} \mathrm{V}$ the $i$ and $f$ lines are rather weak, but for N VI and O VII three clearly separated components were measured. For Mg XI and Si XIII the intercombination line was blended by the resonance line, but a double Gaussian fit enabled us to deconvolve these lines, resulting in measured wavelengths in very good agreement (within about $0.005-0.01 \AA$ ) with the theoretical values. In the case of $\mathrm{Si}$, the measurement should be considered less certain due to the lower spectral resolution at these wavelengths.

From the ratio $G=(i+f) / r$ the temperature is determined for each He-like ion and is given in Table 6 . We observe a wide range of temperatures from about $0.5 \mathrm{MK}$ 
Table 7. Densities from ratios of $n_{\mathrm{e}}$-dependent iron lines

\begin{tabular}{l|cccc}
$\lambda(\AA)$ & $+1^{a}$ & $-1^{a}$ & av. $^{b}$ & $n_{\mathrm{e}}^{c}$ \\
\hline Fe XX & & & & \\
$\frac{110.63}{118.66}$ & 0.15 & 0.16 & $0.15(.06)$ & $<510^{12}$ \\
$\frac{110.63}{121.83}$ & 0.07 & 0.11 & $0.08(.03)$ & $<510^{12}$ \\
$\frac{110.63}{132.85^{d}}$ & 0.07 & 0.07 & $0.04(.02)$ & $<210^{12}$ \\
Fe XXI & & & & \\
$\frac{97.88}{128.74}$ & 0.22 & 0.10 & $0.09(.03)$ & $<210^{12}$ \\
$\frac{117.51}{128.73}$ & 0.34 & 0.20 & $0.17(.05)$ & $<310^{12}$ \\
Fe XXII & & & & \\
$\frac{114.41}{117.17}$ & 0.14 & 0.18 & $0.17(.10)$ & $<310^{12}$ \\
$\frac{114.41}{135.78}$ & 0.30 & 0.33 & $0.19(.10)$ & $<510^{12}$ \\
\hline
\end{tabular}

${ }^{a}$ measured counting rate ratio in +1 and -1 order of spectrum.

${ }^{b}$ line photon flux ratio averaged over +1 and -1 order and corrected for interstellar absorption (in parentheses the mean statistical $1 \sigma$ error).

${ }^{c} n_{\mathrm{e}}\left(\right.$ in $\left.\mathrm{cm}^{-3}\right)$ derived from Brickhouse et al. (1995).

${ }^{d}$ line intensity too high compared to theoretical values (see Table 4 and paragraph 3.2); results less reliable.

for $\mathrm{N}$ VI up to $5 \mathrm{MK}$ for the higher stages of ionization (Si XIII). However, the N VI temperature appears somewhat discrepant in comparison to those derived for $\mathrm{C}$ and $\mathrm{O}$ and we suspect it might be affected by line blending. The temperatures were all derived using an updated version of the plasma radiative loss and line fitting program SPEX developed at SRON and from recent calculations by Porquet \& Dubau (2000) and by Mewe et al. (2001) including all recombinations. Another possibility for obtaining the coronal temperature from He-like ions is to use the ratio between the resonance lines of these ions and the resonance lines of the one higher ionized stages, the H-like ions, of the same element. These ratios and the derived temperatures are collected also in Table 6. The single He-like ion ratios result in a lower temperature than the ratios from He-like versus $\mathrm{H}$-like ions. The conclusion is that the $\mathrm{H}$-like ions are formed in another (hotter) area than the He-like ions. Therefore the obtained temperature is a mixture and average of the two areas.

\section{Density diagnostics}

\subsection{Fe ions}

From a number of density-sensitive iron lines at wavelengths above $90 \AA$ (e.g., Fe XX to Fe XXII) the electron density can be investigated for plasmas with relatively high temperatures $(T \gtrsim 5 \mathrm{MK})$. For the determination of the electron density in the coronal plasma of Capella ratios of density-dependent lines of Fe XX, Fe XXI, and Fe XXII were used. The data used and density values obtained are listed in Table 7. The theoretical density dependence for these lines is taken from the work by Brickhouse et al. (1995). The theoretical flux ratio- $n_{\mathrm{e}}$ curves are very flat
Table 8. Density determination from the $i / f$ line intensity ratio for different He-like ions

\begin{tabular}{l|ccccc}
$\lambda(\AA)$ & $+1^{a}$ & $-1^{a}$ & av. $^{b}$ & $n_{\mathrm{e}}^{c}$ & $n_{\mathrm{e}}^{d}$ \\
\hline C V & & & & & \\
$\frac{40.731}{41.472}$ & sum & & $0.89(.6)$ & $3 \pm 210^{9}$ & $410^{9}$ \\
N VI & & & & & \\
$\frac{29.084}{29.534}$ & 0.52 & 0.53 & $0.49(.17)$ & $5 \pm 310^{9}$ & $610^{9}$ \\
O VII & & & & & \\
$\frac{21.804}{22.101}$ & 0.26 & 0.31 & $0.28(.05)$ & $<710^{9}$ & $310^{9}$ \\
$\begin{array}{l}\text { Mg XI } \\
\frac{9.231}{9.315}\end{array}$ & 0.51 & 0.40 & $0.45(.13)$ & $<410^{12}$ & $310^{12}$ \\
$\begin{array}{l}\text { Si XIII } \\
\frac{6.688}{6.740}\end{array}$ & 0.62 & 0.82 & $0.72(.24)$ & $3 \pm 2.510^{13}$ & $410^{13}$ \\
\hline
\end{tabular}

${ }^{a}$ measured counting rate ratio in +1 and -1 order of spectrum.

${ }^{b}$ line photon flux ratio averaged over +1 and -1 order and corrected for interstellar absorption (in parentheses the mean statistical $1 \sigma$ error).

${ }^{c}$ Electron density (in $\mathrm{cm}^{-3}$ ) derived from SPEX/MEKAL.

${ }^{d}$ Electron density (in $\mathrm{cm}^{-3}$ ) derived from Porquet and Dubau (2000) and Mewe et al. (2001); for C V a radiation temp. of $4585 \mathrm{~K}$ is assumed (see Ness et al. 2001).

for low densities and start to deviate from the low-density plasma situation only for values of $n_{\mathrm{e}} \gtrsim 10^{11} \mathrm{~cm}^{-3}$. This makes the method insensitive for the lower limit. The $n_{\mathrm{e}}$ values obtained for the ions Fe XX to Fe XXII, however, are consistently around $\sim 10^{12} \mathrm{~cm}^{-3}$ and all with an upper limit of $n_{\mathrm{e}} \lesssim 2-510^{12} \mathrm{~cm}^{-3}$.

\subsection{He-like ions}

Two of the three transitions of the He-like ions described above are also strongly density-dependent (e.g., Gabriel \& Jordan 1969), namely the ratio of the intercombination line $(i)$ and the forbidden line $(f)$. Line ratios $(i / f)$ were derived from measured values and the corresponding plasma densities were derived; the results are listed in Table 8 . The uncertainty of the counting rate of the O VII intercombination line justifies an upper limit only. As for the temperature diagnostics, the obtained values are from SPEX and from Porquet \& Dubau (2000) and Mewe et al. (2001).

If we compare the results for $i / f$ and $n_{\mathrm{e}}$ with those obtained by Ness et al. (2001) in the case of C, N, and O, we note that the values are slightly different but in agreement within the error bars. Ness et al. (2001) find for O VII a somewhat different value for $i / f(0.255 \pm 0.015)$ based on a method in which the total spectrum is fitted with a model for the continuum plus background, whereas we fit the background-subtracted spectrum. Because below about $10^{9} \mathrm{~cm}^{-3}$ the dependence of $i / f$ on $n_{\mathrm{e}}$ becomes 
very flat (Fig. 5 of Ness et al.) this gives a much lower upper limit for $n_{\mathrm{e}}$ (only $510^{8} \mathrm{~cm}^{-3}$ ) than we find but the difference is still acceptable within the statistical uncertainties.

From the observations of Capella with the HighEnergy Transmission Grating on CHANDRA Canizares et al. (2000) obtain a slightly larger $i / f$ ratio for O VII $(0.35 \pm 0.05$, but consistent with our ratio of $0.28 \pm$ $0.05)$ implying a higher electron density within the range (8-20) $10^{9} \mathrm{~cm}^{-3}$. Again the difference in $i / f$ may be due to a different treatment of the continuum plus background.

For the lower ions $\mathrm{C} \mathrm{V}, \mathrm{N}$ VI, and O VII we obtain densities typically in the range $10^{9}-10^{10} \mathrm{~cm}^{-3}$ for temperatures between about 0.5 and $2 \mathrm{MK}$. Such densities are typical of those for active coronal regions on the Sun. In the case of Capella, similar densities for the lower temperature $(\sim 1-2 \mathrm{MK})$ plasma were suggested by Schrijver et al. (1995) and by Brickhouse (1996) in analyses of EUVE spectra. The higher ions Mg XI and Si XIII associated with higher temperatures $(z 5 \mathrm{MK})$ suggest much higher densities, i.e. $10^{12}-10^{13} \mathrm{~cm}^{-3}$, that are consistent with the results obtained for the highly ionized Fe ions. Such high densities have been observed on the Sun only during flares, but were derived earlier from EUVE spectra of Capella (cf. Sect. 1). We note, however, that the density obtained for Si XIII is significantly higher than the upper limit found from Mg XI. For the same line complex observed with the CHANDRA HETG (albeit at a different time), Canizares et al. (2000) obtained a ratio $i / f$ of $0.39 \pm 0.03$, which is to be compared with our value of $0.72 \pm 0.24$. It is clear from the recent analysis of HST STIS spectra by Johnson et al. (2001) that the hot 10 MK component of the Capella coronae can be highly variable, and so it is therefore possible that the higher density we derived based on observations made at a different time is real. However, since the spectral resolution of the LETGS is fairly low at the wavelength of the Si He-like complex, rendering line deconvolution more difficult, this density should perhaps be treated with caution at this time.

\section{Emission measure modeling}

We performed an emission measure $(E M)$ analysis of the Capella LETGS data by comparing measured line fluxes with the theoretical fluxes as calculated by MEKAL for a given emission measure and formation temperature for a particular line, assuming solar abundances (Anders \& Grevesse 1989). Figure 4 (top) presents the results of the $E M$ distribution based on the analysis of the iron lines. For all Fe-ions used the error bars are given. These error bars contain two components. When having many lines (and values) for one particular ion, the error is dominated by the statistical error of these values. In case of one single transition or two closely lying values for an ion, the error is dominated by the uncertainty in the flux as given in Tables 1 and 2. The larger error bar for Fe XVI is probably due to the fact that all lines used are near the gap between
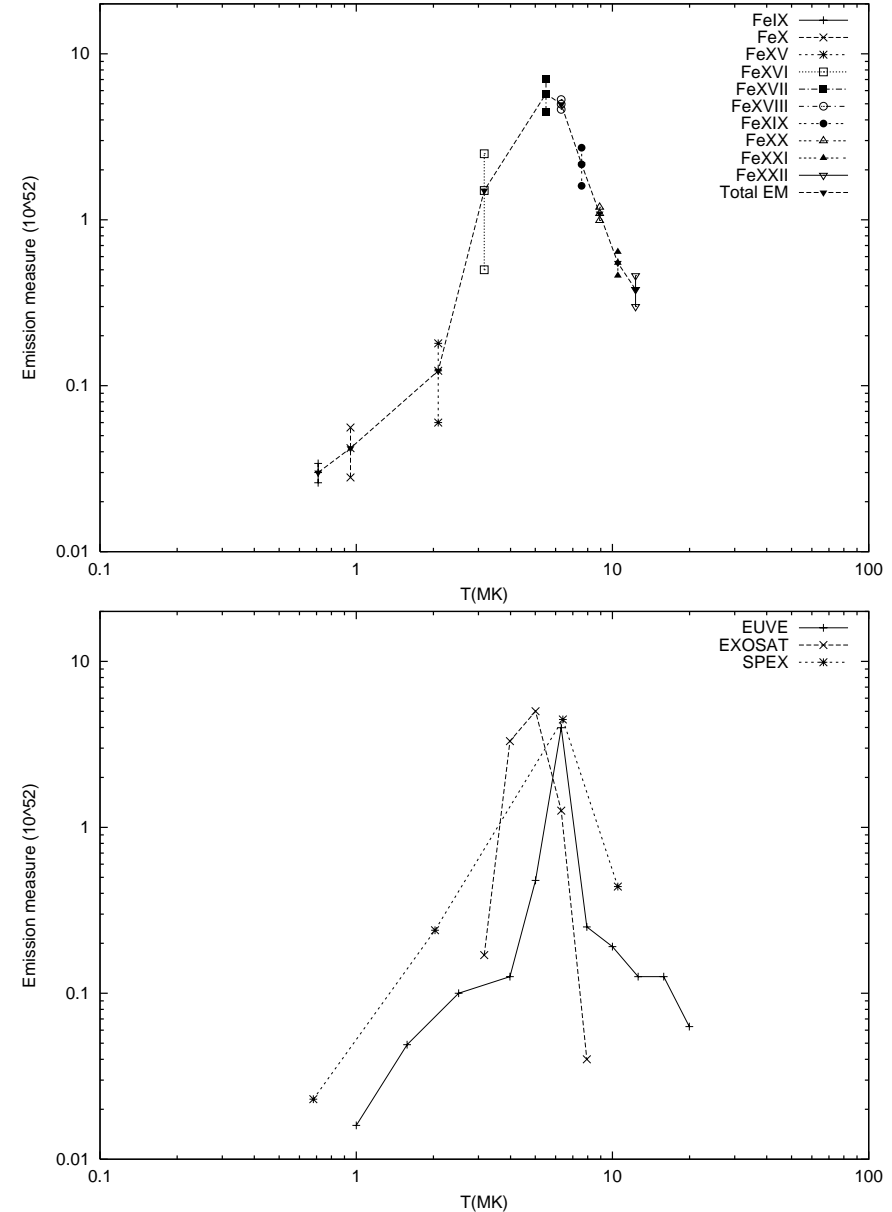

Fig. 3. $E M$ distribution derived from Fe IX to Fe XXII line intensities for the Capella CHANDRA-LETGS observations (top) compared to the results from a $4-T$ fitting with SPEX and from observations with EUVE (Brickhouse et al. 2000) and EXOSAT (Lemen et al. 1989) (bottom)

the detectors where the efficiency declines rapidly and the calibration is uncertain.

For comparison we give in the bottom panel of Fig. 3 the results from two other instruments: EXOSAT (Lemen et al. 1989) and EUVE (Brickhouse et al. 2000). These distributions are comparable to our results given the fact that the results are obtained at different times and with different instruments.

Using the SPEX code (Kaastra et al. 1996) we have fitted the Chandra Capella spectrum with a 4-temperature model with variable abundances. The result scaled to the solar abundance of iron is shown in the bottom panel of Fig. 3 and agrees satisfactorily with the emission measure distribution given on top. The derived ratios of and $\mathrm{Ne}$ to Fe are close to solar or mildly subsolar, while the $\mathrm{N}$, $\mathrm{Mg}$, and Si ratios are about a factor of 2 larger than compared to that taken from Anders \& Grevesse (1989). For $\mathrm{Mg}$ and Si these results agree with those from Brickhouse et al. (2000) based on an analysis of EUVE and ASCA spectra, but our Ne/Fe ratio is a factor of 3 larger. 
Table 9. Characteristic loop parameters derived from observations

\begin{tabular}{l|ccccccc} 
Ion & $n_{\mathrm{e}}^{a}$ & $T_{\mathrm{m}}^{b}$ & $L^{c}$ & $H_{\mathrm{p}}^{d}$ & $E M^{e}$ & $f^{f}$ & $f^{g}$ \\
\hline C V & 0.03 & 1.0 & 4.3 & 1.0 & 0.74 & 73 & 6.5 \\
N VI & 0.05 & 1.4 & 5.1 & 1.4 & 1.10 & 34 & 3.0 \\
O VII & $0.07^{h}$ & 2.0 & 7.4 & 2.0 & 0.71 & 7.4 & 0.68 \\
Mg XI & $40^{h}$ & 6.3 & 0.13 & 6.2 & 6.6 & $1.1^{-2}$ & $1.0^{-3}$ \\
Si XIII & $300^{i}$ & 10 & 0.043 & 9.8 & 5.3 & $5.2^{-4}$ & $4.8^{-5}$ \\
Fe XX & $30^{h}$ & 8.9 & 0.34 & 8.7 & 1.2 & $1.5^{-3}$ & $1.4^{-4}$ \\
Fe XXI & $30^{h}$ & 10 & 0.43 & 9.8 & 0.64 & $6.3^{-4}$ & $5.8^{-4}$ \\
Fe XXII & $30^{h}$ & 12 & 0.62 & 12 & 0.38 & $2.5^{-4}$ & $2.3^{-5}$ \\
\hline
\end{tabular}

${ }^{a}$ electron density at loop apex in $10^{11} \mathrm{~cm}^{-3}$ from SPEX/MEKAL.

${ }^{b}$ temperature at loop apex in MK taken equal to maximum line formation temperature from SPEX/MEKAL.

${ }^{c}$ loop semilength in $10^{8} \mathrm{~cm}$ for $\Gamma=1$; for $\Gamma=10 \mathrm{~L}$ will be factor $\Gamma^{0.3} \simeq 2$ larger; $L$ scales $\propto T_{\mathrm{m}}^{2} / n_{\mathrm{e}}$ and $H_{\mathrm{p}} \propto T_{\mathrm{m}}$.

${ }^{d}$ pressure scale height in $10^{11} \mathrm{~cm}$.

${ }^{e}$ emission measure in $10^{52} \mathrm{~cm}^{-3}$.

${ }^{f}$ filling factor (in \%) for $\Gamma=1 ; 1.1^{-2}=1.110^{-2}$, etc.

${ }^{g}$ filling factor (in \%) for $\Gamma=10 ; 1.0^{-3}=1.010^{-3}$, etc.

${ }^{h}$ to be considered as upper limit, corresponding loop length and filling factor as lower limit.

${ }^{i}$ lower limit is 50 .

\section{Loop modeling}

\subsection{Scaling laws and loop lengths}

Because the two binary components both contribute to the observed X-ray emission (see Sect. 1), any interpretation of the composite spectrum can only be given in terms of average stellar properties. We derive from our observations global properties of the X-ray emitting coronal structures using a standard model of a quasi-static, semi-circular, magnetic loop with constant cross section as developed by Rosner et al. (1978; hereafter RTV). In the RTV loop model the basic loop parameters are related through a simple scaling law:

$T_{\mathrm{m}} \simeq 1400(p L)^{1 / 3}$,

where the apex temperature $T_{\mathrm{m}}$ in $\mathrm{K}$, the loop semilength $L$ in $\mathrm{cm}$, and the pressure $p$ in dyne $\mathrm{cm}^{-2}$. Or with $p \simeq$ $2 n_{\mathrm{e}} k T_{\mathrm{m}}$ we write:

$n_{\mathrm{e}} L=1.310^{6} T_{\mathrm{m}}^{2}$,

where $n_{\mathrm{e}}$ is the apex electron density in $\mathrm{cm}^{-3}$.

Generalizations of the basic RTV model have been developed by Vesecky et al. (1979; hereafter VAU), including cross sections that increase with height in a way representing loop field structures by a magnetic line dipole below the chromosphere. The scaling law is only slightly modified by the VAU results which can be conveniently approximated by introducing into the rhs of Eqs. (3 or 4) an additional multiplication factor $\Gamma^{-0.1}$ or $\Gamma^{0.3}$, respectively. Here $\Gamma$ is the ratio of the loop cross section at the apex to that at the loop footpoint, the expansion factor. It turns out that a large variety of solar loops ranging from small bright points to active-region loops and large-scale structures can be modeled by these scaling laws (e.g., discussion by Mewe 1991).

Applying Eq. (4) for the derived densities (Sect. 5) we obtain estimates for the loop semilengths $L$ (Table 9), taking $T_{\mathrm{m}}$ equal to the temperature of maximum line formation (from MEKAL).

\subsection{Emission measures and filling factors}

For a given density the emission measure places an additional constraint on the emitting volume (i.e. the "filling factor"). From the measured line fluxes and the known emissivities at temperature of maximum line formation we derive the emission measure $E M=\int n_{\mathrm{e}} n_{\mathrm{H}} \mathrm{d} V$, where $V$ is the volume contributing to the emission (for cosmic abundances the hydrogen density $n_{\mathrm{H}} \simeq 0.85 n_{\mathrm{e}}$ ). In Table 9 we give $E M$ for various lines.

Assuming for simplicity that the X-ray corona consists of one class of $N$ identical loops with parameters $T_{\mathrm{m}}, n_{\mathrm{e}}, L$, we derive for the emission measure

$E M=0.85 G C(\Gamma) f n_{\mathrm{e}}^{2}\left(4 \pi R^{2}\right) \ell$,

where $R$ is the stellar radius $\simeq 10 R_{\odot} \simeq 710^{11} \mathrm{~cm}$ (Strassmeier et al. 1993) averaged for the two components of Capella. The factor $G$ corrects for the fraction of the corona occulted by the stellar disk: $G=0.5$ for compact loops which we consider here. $C(\Gamma) \simeq(1+\Gamma) / 2$ is an empirical correction for $\Gamma>1$ to the RTV loop volume derived from the VAU model. The fractional coverage of the stellar surface with footpoints of $N$ identical X-ray emitting loops with cross section $S$ at the base, the socalled filling factor, equals $f=2 N S / 4 \pi R^{2}$. Finally, $\ell$ is the emission scale length, which is limited either by the loop size $\left(\ell \approx L\right.$ for $\left.L<H_{\mathrm{p}} / 2\right)$ (case 1 ) or by the pressure scale height $\left(\ell \approx H_{\mathrm{p}} / 2\right.$ for $\left.L>H_{\mathrm{p}} / 2\right)$ (case 2$)$. For case 1 (as $L \ll H_{\mathrm{p}}$ ) we derive from Eq. (6) and using Eq. (4) (with the factor $\Gamma^{0.3}$ ) for the filling factor

$f=1.4510^{-7} \frac{E M}{n_{\mathrm{e}} R^{2} T_{m}^{2} C(\Gamma) \Gamma^{0.3}}$.

Using the measured fluxes for various helium-like lines and some iron lines we present in Table 9 the filling factors calculated with Eq. (7) for two loop geometries: $\Gamma=1$ (RTV loop) and $\Gamma=10$, respectively. The filling factors for the low-temperature regions are about $30 \%$ or more for the case $\Gamma=1$, i.e., comparable to those for active RS CVn systems, but much higher than for active regions on the Sun (few \%, cf. Mewe 1991). However, loops with large expansion $(\Gamma=10)$ should have a filling factor that is closer to that of active regions on the Sun. The hot, compact loops have probably a very small filling factor.

The results appear to show the existence of two main classes of loop structures in the coronae of the binary system Capella. On the one hand, the analysis of the lower ions N VI and O VII indicates temperatures, densities, 
and loop lengths that are more or less representative of active coronal regions on the Sun (with relatively large filling factors).

On the other hand, the higher He-like ions Mg XI and Si XIII and the highly ionized Fe ions (Fe XX-XXII) indicate the presence of a class of much more compact and dense, hot loops (with very small filling factors). On the Sun temperatures and densities this high - as observed in non-flaring active regions in the coronae of Capella - are seen only during flares. For both classes of loop systems $L \ll H_{\mathrm{p}}$ and $R$, where the pressure scale height $H_{\mathrm{p}}$ (in $\mathrm{cm})$ is given by

$H_{\mathrm{p}}=510^{3} T\left(g / g_{\odot}\right)^{-1}$,

where $g / g_{\odot}$ is the surface gravity expressed in solar units (for Capella $=0.051$ ) and $T$ in $\mathrm{K}$.

In all cases the loop sizes are much smaller than the stellar radius so that it is clear that the X-ray emission originates from compact regions that must be supported by strong magnetic fields (e.g., of the order of several hundred Gauss as was suggested by Dupree et al. 1993). Similar conclusions were drawn for other active binaries based on a review of EUVE observations by Drake (1996).

\section{Conclusions}

Echo the salient features of earlier EUVE analyses, we have established different temperature regimes in the Capella coronal plasmas: from $T \sim 0.5-2 \mathrm{MK}$ for Fe IX $\& \mathrm{X}, \mathrm{N}$ VI, and O VII, $5-6$. MK for Fe XVIII-XX up to $\sim 9 \mathrm{MK}$ for Fe XXI and Fe XXII. The upper limit to the densities obtained from Fe XX-XXII lines is about $310^{12} \mathrm{~cm}^{-3}$. The density of the plasma obtained from density sensitive lines of He-like triplets N VI and O VII is in the range $10^{9}-10^{10} \mathrm{~cm}^{-3}$ for $T \simeq 0.5-2 \mathrm{MK}$ and from Mg XI and Si XIII in the range $10^{12}-10^{13} \mathrm{~cm}^{-3}$ for $T \simeq 5-9 \mathrm{MK}$.

Abundances derived for various elements are about so$\operatorname{lar}(\mathrm{O}, \mathrm{Ne})$ or twice solar $(\mathrm{N}, \mathrm{Mg}, \mathrm{Si})$.

The results for the densities and emission measures are interpreted in terms of quasi-static loop models. They appear to show the existence of two main classes of loop structures in the coronae of Capella. The analysis of the lower ions N VI and O VII indicates temperatures, densities, and loop lengths that are more or less representative for active coronal regions on the Sun (with relatively large filling factors). On the other hand, the higher Helike ions Mg XI and Si XIII and the highly ionized Fe ions (Fe XX-XXII) indicate the presence of a class of much more compact and dense, hot loops (with very small filling factors). On the Sun temperatures and densities this high are seen only during flares. For both classes of loops the size is much smaller than the pressure scale height and the stellar radius.

Comparisons between ratios of lines decaying from the same upper level and branching ratios give confidence in the in-flight total area calibration and the detector sensitivity as well as the reliability of the calculated transition probabilities. Based on photon fluxes of lines, sensitive to resonance scattering, we have derived conservative upper limits to the optical depth of the lines Fe XVIII $\lambda 93.92$ and Fe XVII $\lambda 15.014$ of 1 and 1.5, respectively.

Acknowledgements. The Space Research Organization Netherlands (SRON) is supported financially by NWO. We thank Nancy Brickhouse for her contribution to the effort to obtain for Capella a long observation time with the LETGS during the initial in-flight calibration of the CHANDRA transmission gratings in the context of the "Emission Line Project" headed by her and Jeremy Drake. JJD was supported by NASA contract NAS8-39073 to the Chandra X-ray Center during the course of this research. Finally, we wish to thank the referee for helpful comments that allowed us to improve the paper.

\section{References}

Anders, E., \& Grevesse, N. 1989, Geochimica et Cosmochimica Acta, 53, 197

Arnaud, M., \& Raymond, J. 1992, ApJ, 398, 394

Audard, M., Behar, E., Güdel, M., et al. 2001, A\&A, in press

Bhatia, A. K., \& Doschek, G. A. 1992, At. Data Nucl. Data Tables, 52, 1

Brickhouse, N. S. 1996, in Proc. IAU Coll. 152, Astrophysics in the Extreme Ultraviolet, ed. S. Bowyer, \& R. F. Malina (Dordrecht, Kluwer), 105

Brickhouse, N. S., Raymond, J. C., \& Smith, B. W. 1995, ApJS, 97,551

Brickhouse, N. S., Dupree, A. K., Edgar, R. J., et al. 2000, ApJ, 530, 387

Brinkman, A. C., Gunsing, C. J. T., Kaastra, J. S., et al. 2000, ApJL, 530, L111

Brown, G. V., Beiersdorfer, P., Liedahl, D. A., et al. 1998, ApJ, 502,1015

Canizares, C. R., Huenemoerder, D. P., Davis, D. S., et al. 2000, ApJL, 539, L41

Cowan, R. D. 1981, The Theory of Atomic Structure and Spectra (Berkeley, CA, Univ. of California Press)

Drake, J. J. 1996, in Cool Stars, Stellar Systems and the Sun, Ninth Cambridge Workshop, ed. R. Pallavicini, \& A. Dupree, ASP Conf. Ser., 109, 203

Dupree, A. K., Brickhouse, N. S., Doschek, G. A., Green, J. C., \& Raymond, J. C. 1993, ApJ, 418, L41

Gabriel, A. H., \& Jordan, C. 1969, MNRAS, 145, 241

Johnson, O., Drake, J. J., Kashyap, V., et al. 2001, submitted to ApJL

Kaastra, J. S., \& Mewe, R. 1995, A\&AL, 302, L13

Kaastra, J. S., Mewe, R., \& Nieuwenhuijzen, H. 1996, in UV and X-ray Spectroscopy of Astrophysical and Laboratory Plasmas, ed. K. Yamashita, \& T. Watanabe (Universal Academy Press, Inc., Tokyo), 411 (SPEX)

Lemen, J. R., Mewe, R., \& Schrijver, C. J. 1989, ApJ, 341, 474

Linsky, J. L., Wood, B. E., Brown, A., \& Osten, R. A. 1998, ApJ, 492, 767

Mason, H. E., Bhatia, A. K., Kastner, S. O., Neupert, W. M., \& Swartz, M. 1984, Solar Phys., 92, 199

Mewe, R. 1991, Adv. Space Sci. Rev., 11, (1) 127

Mewe, R., Gronenschild, E. H. B. M., \& van den Oord, G. H. J. 1985, A\&ASS, 62, 197

Mewe, R., Kaastra, J. S., \& Liedahl, D. A. 1995a, Legacy, 6, 16 (MEKAL) 
Mewe, R., Kaastra, J. S., Schrijver, C. J., van den Oord, G. H. J., \& Alkemade, F. J. M. 1995b, A\&A, 296, 477

Mewe, R., Lemen, J. R., \& Schrijver, C. J. 1991, Astrophys. Space Sci., 182, 35

Mewe, R., Porquet, D., Raassen, A. J. J., \& Dubau, J. 2001, in preparation

Morrison, R., \& McCammon, D. 1983, ApJ, 270, 119

Ness, J.-U., Mewe, R., Schmitt, J. H. M. M., et al. 2001, A\&A, in press

Phillips, K. J. H., Mewe, R., Harra-Murnion, L. K., et al. 1999, A\&ASS, 138, 381

Porquet, D., \& Dubau, J. 2000, A\&ASS, 143, 495

Rosner, R., Tucker, W. M., \& Vaiana, G. S. 1978, ApJ, 220, $643(\mathrm{RTV})$
Saba, J. L. R., Schmelz, J. T., Bhatia, A. K., \& Strong, K. T. 1999, ApJ, 510, 1064

Schrijver, C. J., Mewe, R., van den Oord, G. H. J., \& Kaastra, J. S. 1995, A\&A, 302, 438

Strassmeier, K. G., Hall, D. S., Fekel, F. C., \& Scheck, M. 1993, A\&ASS, 100, 173

van der Meer, R. L. J., et al. 2001, in preparation (calibration paper)

Vesecky, J. F., Antiochos, J. H., \& Underwood, J. H. 1979, ApJ, 233, 987 (VAU)

Wood, K., \& Raymond, J. 2000, ApJ, 540, 563 